\title{
Criminologie
}

\section{Nouvelles politiques publiques belges sécuritaires et sociosanitaires en matière de toxicomanie : à la recherche d'une logique d'alliance...}

\section{Marie-Sophie Devresse et Jean-François Cauchie}

Volume 33, numéro 2, automne 2000

Problèmes sociaux et système pénal

URI : https://id.erudit.org/iderudit/004717ar

DOI : https://doi.org/10.7202/004717ar

Aller au sommaire du numéro

Éditeur(s)

Les Presses de l'Université de Montréal

ISSN

0316-0041 (imprimé)

1492-1367 (numérique)

Découvrir la revue

Citer cet article

Devresse, M.-S. \& Cauchie, J.-F. (2000). Nouvelles politiques publiques belges sécuritaires et sociosanitaires en matière de toxicomanie : à la recherche d'une logique d'alliance... Criminologie, 33(2), 109-117.

https://doi.org/10.7202/004717ar
Résumé de l'article

En Belgique comme ailleurs en Europe, les années 1990 auront symbolisé le retour au premier plan du thème de l'insécurité, et ce, notamment dans le domaine de la toxicomanie. Une préoccupation qui allait notamment se traduire par une volonté politique de créer une alliance entre intervenants de terrain défendant des projets à vocation " sociale » et des acteurs porteurs de projets à connotation socio-sécuritaire ou policière. À cet égard, cet article entend présenter le point de vue de ces différents acteurs et montrer comment l'imposition d'une dynamique d'alliance a conduit ceux-ci à adopter des positions de repli ou de frileuse collaboration. Notre réflexion entendra dès lors s'interroger sur les places respectives des approches pénales et sociales en la matière, ainsi que de produire une réflexion critique sur l'évolution du recours à la réponse répressive en général. 


\title{
Nouvelles politiques publiques belges sécuritaires et sociosanitaires en matière de toxicomanie : à la recherche $d^{\prime}$ une logique $d^{\prime}$ alliance... ${ }^{1}$
}

\author{
Marie-Sophie Devresse \\ Assistante et chercheuse \\ Département de criminologie et de droit pénal \\ Université Catholique de Louvain - Belgique \\ devresse@crim.ucl.ac.be \\ Jean-François Cauchie \\ Aspirant F.N.R.S. \\ Département de criminologie et de droit pénal \\ Université Catholique de Louvain - Belgique \\ cauchie@crim.ucl.ac.be
}

RÉSumÉ - En Belgique comme ailleurs en Europe, les années 1990 auront symbolisé le retour au premier plan du thème de l'insécurité, et ce, notamment dans le domaine de la toxicomanie. Une préoccupation qui allait notamment se traduire par une volonté politique de créer une alliance entre intervenants de terrain défendant des projets à vocation " sociale » et des acteurs porteurs de projets à connotation socio-sécuritaire ou policière. À cet égard, cet article entend présenter le point de vue de ces différents acteurs et montrer comment l'imposition d'une dynamique d'alliance a conduit ceux-ci

1. Les réflexions présentées dans cet article sont issues des résultats d'une recherche interuniversitaire financée par les Services fédéraux des Affaires scientifiques, techniques et culturelles du gouvernement belge. Cette recherche, étendue sur une période de quatre ans, a impliqué quatre équipes de recherche : le Centre d'Études Sociologiques des Facultés Universitaires Saint-Louis de Bruxelles, l'Unité de recherche en criminologie de l'Université Catholique de Louvain (à laquelle appartiennent les auteurs), l'École des sciences criminologiques Léon Cornil de l'Université Libre de Bruxelles ainsi que l'association sans but lucratif bruxelloise « Synergie».

Criminologie, vol. 33, $n^{\circ} 2$ (2000) 
à adopter des positions de repli ou de frileuse collaboration. Notre réflexion entendra dès lors s'interroger sur les places respectives des approches pénales et sociales en la matière, ainsi que de produire une réflexion critique sur l'évolution du recours à la réponse répressive en général.

RÉSUMÉ - In Belgium as elsewhere in Europe, the 1990's will have symbolized refocusing on the insecurity debate, in particular in the field of drug addiction: a concern that was, among others, expressed by the political will to create an alliance between practitioners defending "social" projects and those who support security or policing programs. In this respect, this article presents, on the one hand, the point of view of these different persons, and on the other hand, demonstrates how the imposition of alliance dynamics drove them to adopt positions of withdrawal or dubious collaboration. Our research then questions the respective places of penal and social approaches to this problem, and proposes a critical perspective of the evolution of a recourse to the repressive solution in general.

Avec la période des angoisses ou des réjouissances « fin de siècle » vient aussi inévitablement la période des bilans. Parmi ceux que la Belgique pourrait réaliser en matière pénale, nous avons opté aujourd'hui pour une approche critique des «politiques» successives qu'elle a connues durant la dernière décennie en matière de prévention, de sécurité et de justice. À l'intérieur de ces politiques, des objectifs déclarés et d'autres moins ouvertement affirmés. En tout cas, des velléités de repenser autrement l'administration de la justice criminelle à tous ses niveaux, par le biais de l'introduction de nouveaux dispositifs ou par l'arrivée de nouveaux acteurs situés à la frontière de la sphère pénale et de la sphère sociale.

\section{Un pays marqué par une politique pénale en mutation}

Le début des années 1990 en Belgique est marqué en effet par une profusion de projets qui tentent, à leur façon, d'interroger la question pénale. Ces projets, que nous allons tout d'abord présenter succinctement, semblent à priori relativement diversifiés mais participent de concert à un mouvement qui se veut réformateur. Dans des domaines aussi divers que la sécurité du citoyen, l'accélération de la justice pénale, ou encore la réforme des polices, la Belgique a singulièrement fait preuve de dynamisme, non seulement par sa volonté de faire face concrètement à des événements tragiques, mais aussi par une ferme détermination à répondre à l'angoisse suscitée par ceux-ci dans la popu- 
lation. C'est que, avant la tristement célèbre «affaire Dutroux », le plat pays s'était déjà illustré par d'autres événements plus ou moins dramatiques $^{2}$ dont on est en droit de penser qu'ils ont directement conduit les autorités fédérales à faire œuvre de création dans le domaine des politiques publiques.

À l'intérieur de ces politiques, un fil conducteur : la volonté de travailler à la sécurisation du citoyen par de nouvelles formes de réponses. Un objectif multiforme qui se décline en une multiplicité de priorités : restaurer la confiance du citoyen dans les autorités publiques (policières, judiciaires et politiques) $^{3}$, et donc, travailler entre autres à l'efficacité de l'ensemble du dispositif de prévention et de répression, restaurer le « lien social » en favorisant la mise en place de nouvelles procédures de médiation ou de nouveaux lieux de rencontre institutionnels, et surtout optimaliser ou renforcer l'emploi des ressources existantes, à tout le moins en ce qui concerne l'appareil judiciaire et policier.

L'ensemble de ces objectifs participe à la réalisation d'un vaste projet entendant faire face à ce que l'on pourrait qualifier de « crise » (Cartuyvels et Mary, 1997 : 97) des appareils de régulation et de contrôle. Une crise dont les autorités fédérales ont cru lire les effets dans les diverses manifestations de désintérêt du citoyen face à la politique gouvernementale et aux institutions de son pays... Le problème posé est donc avant tout politique et prend la question pénale comme tremplin pour assurer la " relance », sinon d'une affiliation citoyenne tout au moins d'une vague de recrutement à visée électoraliste, les autorités publiques ne s'étant pas cachées de voir la mise au travail de centaines d'intervenants sociaux comme un objectif en soi.

Avant de nous interroger sur les présupposés à l'œuvre dans ce type de choix politique et institutionnel, nous allons plus particulièrement examiner deux des dispositifs les plus représentatifs de ces réformes.

2. A peine remise du traumatisme suscité par les actes — non élucidés — de grand banditisme de la fin des années 1980 (« les tueries du Brabant »), la Belgique a vu la une de ses journaux envahie successivement par la cavale meurtrière de deux individus qualifiés un peu rapidement de «toxicomanes » ainsi que par les feux des « mini-émeutes » bruxelloises de deux quartiers populaires à forte densité immigrée.

3. Communication gouvernementale du 5 juin 1990 relative au «Plan de Pentecôte » du gouvernement fédéral. Annales parlementaires, Chambre des représentants, 56, 2587. 


\section{Deux dispositifs innovants}

Une politique urbaine de prévention

et de sécurité : les contrats de sécurité

Une première étape caractéristique de ce mouvement d'ensemble est celle de la création d'une véritable politique de la ville dont l'aboutissement se voit aujourd'hui confirmé par la naissance, dans notre nouveau gouvernement, du poste de "commissaire chargé de la politique de la ville ». Cette politique, mise en place de façon progressive au départ d'initiatives locales et rapidement coordonnée par le ministère de l'Intérieur et les institutions régionales, s'illustre par les désormais célèbres " contrats de sécurité ». Ces contrats spécifiques, conclus dès 1992 par les deux instances précitées avec les villes et communes belges considérées comme étant «à risques », s'inscrivent dans le cadre plus global d'un « nouveau contrat avec le citoyen » dont l'objectif est « d'apporter une réponse à cinq grands défis sociaux : la sécurité du citoyen, plus d'équité pour une meilleure administration de la justice, une meilleure maîtrise de l'immigration, une politique de l'environnement plus poussée, une société solidaire $»^{4}$.

Le principe des contrats locaux conclus avec les villes et communes est assez simple et emprunte aux concepts de « contrats de ville » de nos voisins français : d'un côté les autorités fédérales et régionales fournissent aux acteurs communaux les moyens nécessaires (issus d'un « fonds de sécurité » voté au sein du budget gouvernemental) pour assurer la sécurité de leurs administrés, de l'autre, ces autorités locales s'engagent à mettre en œuvre divers projets en vue d'assurer concrètement cette sécurité, notamment par des programmes de prévention de la délinquance, ainsi qu'un renforcement financier des dispositifs de police communale.

En termes de politique publique, le choix en Belgique du modèle contractuel semblait à l'époque assez novateur : « la contractualisation avait été pensée en vue de tenir compte des spécificités locales et de répondre aux besoins exprimés par la population, la commune étant vue comme l'instance la plus proche des citoyens et la plus apte à cerner ses aspirations » (Devresse, $1998: 20$ ). Chaque contrat se devait par ailleurs d'être négocié dans la plus pure tradition juridique. Les villes devaient proposer un projet construit qui allait comporter la création de divers

4. Déclaration gouvernementale du 9 mars 1992, Sénat de Belgique, compte rendu analytique, 9 mars 1992, 34. 
services et l'engagement de personnel approprié. Les ministères fédéral et régionaux devaient alors marquer ou non leur approbation aux projets faisant l'objet d'un financement de leur part.

Ainsi, en termes de dispositifs et de projets, la contractualisation allait avoir pour effets de diversifier les initiatives, chaque entité faisant le choix de privilégier tel ou tel domaine (y compris la prévention de la toxicomanie), démontrant ou non de la créativité dans ses projets mis en œuvre, privilégiant telle ou telle conception de la prévention ou de la sécurité, etc. Parler d' « une » nouvelle politique de prévention en Belgique n'a donc de sens que si l'on entend définir, par l'entremise de ces contrats, les velléités interventionnistes du ministère de l'Intérieur et des institutions régionales via la promotion d'une action concertée avant tout « globale et intégrée » sur laquelle nous reviendrons. Notons toutefois dès à présent un élément caractéristique de cette politique, à savoir la répartition des financements en deux volets distincts, celui des projets à connotation policière et celui des projets à vocation dite "sociale » dans lesquels sera particulièrement valorisé l'objectif générique de sécurisation par la prévention.

Un autre effet patent de cette politique allait consister en l'arrivée, sur ce terrain de la prévention, d'un grand nombre de travailleurs sociaux d'un nouveau type ${ }^{5}$, c'est-à-dire de personnes engagées et mises directement sous l'autorité des pouvoirs publics, les projets étant gérés par un organe du ministère de l'Intérieur. Des travailleurs sociaux, dont la difficile tâche allait notamment consister à se faire une place à l'intérieur d'un secteur déjà occupé par d'autres intervenants ${ }^{6}$ quant à eux plus faiblement subventionnés par l'État et dont le travail, bien que la plupart du temps orienté vers un objectif non sécuritaire, allait se trouver engagé à contrecœur dans une sorte de « concurrence » (mêmes publics, mêmes terrains géographiques d'intervention, activités parfois identiques, etc.). Il ne fut donc pas étonnant de voir l'avènement des contrats de sécurité s'accompagner de multiples contestations et débats, portant notamment sur le fait de savoir quelle allait être la liberté d'action, ainsi que les

5. Parmi ceux-ci, nous désignerons désormais les intervenants travaillant dans le secteur de la toxicomanie par l'expression nouveaux intervenants MPS.

6. Nous avons pris l'option de désigner désormais ces intervenants par l'expression travailleurs du secteur traditionnel. Le choix du terme « traditionnel » n'est pas sans soulever un certain paradoxe, dans la mesure où, depuis les années 1970, ces travailleurs revendiquent le plus souvent une identité militante et l'emploi de modèles d'intervention plutôt non conformistes. Il s'agit de travailleurs indépendants issus du secteur privé et bénéficiant pour la plupart de subventions publiques relativement sommaires. 
présupposés éthiques et théoriques sur lesquels allait reposer le travail des nouveaux arrivés.

Une politique de réduction des risques dans le secteur de la toxicomanie : le programme d'action gouvernemental « toxicomanie-drogues»

Dans le domaine particulier de la «toxicomanie », ou plus précisément de « l'usage de drogues », ces mêmes années de remous politiques furent marquées par une inflation de dispositions en tout genre ${ }^{7}$. En 1995, l'arrivée d'un Programme d'action toxicomanie-drogues ${ }^{8}$ allait semer un véritable trouble dans les esprits des travailleurs médico-psycho-sociaux de ce secteur, déjà échaudés par la création récente d'un centre résidentiel dénommé Transit, financé par les contrats de sécurité et dont l'ambition était de prendre en charge sur le mode socio-sanitaire les usagers de drogues interpellés par les forces de l'ordre.

Le programme d'action gouvernemental poursuivait comme principal objectif la promotion d'une politique générale et transversale de réduction des risques pour la santé et pour la criminalité spécifique. Pour y accéder, dominait le mot d'ordre de décloisonnement entre les différents types d'initiatives dans le domaine de la toxicomanie. Mais alors qu'on pourrait trouver dans ce décloisonnement la marque d'une nouvelle approche, voire d'une nouvelle culture résolument non répressive de la question des usages de drogues, on est en droit de faire preuve d'un certain scepticisme à la lecture du texte même du plan gouvernemental.

Ainsi, parmi les dix points énoncés par celui-ci, la mise en place de maisons d'accueil sociosanitaires gérées par le ministère de l'Intérieur incarne sans doute de façon la plus évidente les résolutions sécuritaires poursuivies par le programme gouvernemental à travers le concept de réduction des risques. «L'objectif de ces structures, dont le personnel en majorité médical est habilité à prescrire et administrer des thérapies de substitution, est de toucher le public des usagers de drogue les moins demandeurs, cela notamment afin de diminuer les comportements délinquants de la population toxicomane. La séquence "toxicomanie cause d'insécurité » trouve là une place de choix et jette les bases d'une poli-

7. Nous ne présenterons ici que les plus importantes, en notant le fait qu'aucune forme de dépénalisation ou de légalisation ne fut adoptée en Belgique durant cette période, malgré le dépôt de divers projets ou propositions de loi poursuivant l'une ou l'autre de ces ambitions.

8. Programme d'action du gouvernement fédéral, Conseil des ministres, 3 février 1995, non publié. 
tique uniformisée (à la différence de la diversification caractéristique des contrats de sécurité) où la visée répressive prend le dessus, dans une logique qui relègue la dépénalisation à l'arrière-plan » (Devresse, 1996 : 44). Un tel dispositif fédéral se fonde sur un échec présupposé (jusqu'ici non démontré) des politiques sectorielles de soin, de prévention menées antérieurement ${ }^{9}$ dans le domaine de la toxicomanie par les acteurs «traditionnels » du secteur. Un présupposé qui permet au passage d'attribuer au ministère de l'Intérieur le quasi-monopole des compétences fédérales en matière de toxicomanie, ce qui peut paraitre pour le moins surprenant. ${ }^{10}$

\section{L'usage de drogues : gestion pénale ou gestion "alternative "?}

Un intérêt pour des acteurs en situation

Les nouvelles politiques publiques que nous venons de décrire allaient produire toute une série d'effets à l'intérieur des champs pénal et social. Pour illustrer ceux-ci, nous retiendrons la question de l'usage de drogues. Cette matière permet en effet d'appréhender diverses logiques allant de la plus répressive à la plus libérale et de rendre compte des diverses « tendances » qui ont cours à l'heure actuelle dans notre pays en termes de criminalisation - décriminalisation. Notre réflexion entendra s'interroger sur les places respectives des approches pénales et sociales en la matière, ainsi que de produire une réflexion critique sur l'évolution du recours à la réponse répressive en général.

À cet égard, la position respective des travailleurs sociaux des diverses obédiences identifiées ci-avant, leur identité, leurs représentations, leur position institutionnelle ou leur mandat constituent d'excellents analyseurs des diverses logiques inspirant les mouvements de réforme que nous avons observés. Ainsi, nous n'examinerons pas le contenu proprement dit des transformations proposées, ni les effets de leur mise en œuvre au regard des objectifs qu'elles poursuivent. Nous choisirons plutôt d'examiner leurs effets de configuration ou re-configuration du secteur d'intervention à l'intérieur desquelles elles s'inscrivent. Il nous semble en effet qu'une attention portée aux acteurs sur lesquels repose

9. Voyez plus particulièrement Cartuyvels (1996a).

10. En effet, on peut s'étonner de constater l'absence du ministère de la Santé publique dans l'ensemble des négociations ayant initié les projets mentionnés. 
la mise en œuvre d'une politique dite " globale et intégrée », bien que représentant une approche « oblique » de la question, en dit bien plus long que l'examen des diverses phases de sa mise en œuvre.

\section{Des velléités d'alliance}

L'ensemble de ces nouvelles politiques publiques entendaient donc imposer l'adoption d'une démarche globale intégrée, soit une approche qui réunit tous les acteurs concernés par une problématique. C'est notamment le cas en ce qui concerne la problématique de la toxicomanie; une problématique où devaient se rencontrer les acteurs politiques, les policiers communaux et les intervenants médico-psycho-sociaux. ${ }^{11}$

Pourquoi s'inscrire dans une approche globale intégrée ? Une approche globale d'abord. Nous pourrions, avec Kaminski, l'opposer à une approche totale. Par approche totale, celui-ci entend «le recours à des réponses universelles (valables pour tous) et totalisantes qui s'épuisent aujourd'hui tant dans leur efficacité que dans leur idéologie. La pratique montre en effet qu'aucune corporation ne peut plus prétendre, sans s'associer dans un réseau d'intervenants multiples, traiter définitivement un problème social aux facettes aussi diverses que la toxicomanie » (Kaminski, 1996 : 233). Au constat de « cette incomplétude des stratégies sectorielles ou corporatrices (psychothérapeutiques, policières, médicales, sociales) répondrait alors une approche globale ouvrant à la perspective de se rencontrer [ faire alliance »], au mieux pour se reconnaitre dans sa pertinence sectorielle, au pire, pour se complémenter et (faire) croire qu'ensemble, on reconstitue une nouvelle totalité » (Kaminski, 1996 : 234). Quant à l'approche intégrée, elle prétend rassembler autour des projets locaux tous les acteurs dont la participation serait appropriée pour une politique multidisciplinaire de prévention.

$\mathrm{Si}$, d'un point de vue théorique, l'adoption d'une approche globale intégrée paraît séduisante, le terrain rend compte d'une tout autre réalité. Les représentations et les pratiques des acteurs concernés (les policiers communaux, les intervenants MPS «traditionnels » et nouveaux) laissent en effet entendre qu'il y aurait un fossé entre discours et empirie. Les réflexions suivantes sont issues de l'exploitation d'un matériel de recherche obtenu par entretiens semi-directifs. Elles distinguent les points de vue de trois catégories d'acteurs concernant l'avènement des nouvelles politiques publiques en matière de toxicomanie et

11. Ci-après dénommés « MPS ». 
mettent en lumière la dynamique d'alliance qu'elles ambitionnent de créer sur le terrain entre les différents intervenants.

Secteur « traditionnel »

Désireux de se distinguer des « nouveaux » intervenants MPS en toxicomanie, la plupart des intervenants « traditionnels » interrogés rappellent que leur finalité est socio-sanitaire et non sécuritaire. En d'autres termes, là où ils voient leurs nouveaux concurrents faire du sécuritaire sous couvert de socio-sanitaire, ils défendent pour leur part des objectifs « sociaux » (responsabilisation, émancipation, mise en place de solidarités actives, etc.) et ce, indépendamment des effets sécuritaires que leur action peut engendrer.

Quelle confiance accorder alors aux nouveaux intervenants MPS dont le « sécuritaire » serait l'objectif principal ? La logique d'action de ces intervenants sera-t-elle indépendante de l'autorité qui les finance? Deux questions que semblent se poser régulièrement les intervenants « traditionnels » à propos de leurs nouveaux vis-à-vis. Si la plupart des membres du secteur « traditionnel » s'accordent à juger contraignant le cadre de leurs nouveaux collègues, ils divergent par contre dans la manière de réagir face à ceux-ci. Ainsi en est-il de la question de la formation permanente des nouveaux arrivés. Pour les uns, elle est nécessaire à ces intervenants afin de prendre distance par rapport à leur cadre " sécuritaire »; pour les autres, ce cadre reste une trop forte contrainte, et rend toute formation « sociale » inefficace.

Pour le secteur « traditionnel », les contrats de sécurité représentent en outre une concurrence financière déloyale ainsi qu'une concurrence idéologique inquiétante (se traduisant par un rejet du « toxicomane » via une importante diffusion de méthadone et un retour à l'idéal d'abstinence).

Aux yeux des intervenants "traditionnels", ces contrats engendrent non seulement des conflits de compétence mais consacrent également un retour au vertical. Alors que ces contrats prétendaient, à l'instar de toute la politique sociale des années 1980, privilégier la créativité du secteur, en concertation et en alliance avec les pouvoirs locaux, ils amèneront au contraire les élus locaux, souvent peu motivés ou imaginatifs, à prendre sous leur tutelle des projets imaginés « en haut », par le ministère de l'Intérieur. Des projets qui, rappelons-le, "prétendront associer, dans une visée de "partenariat", répression pénale, prévention policière et prévention sociale » (Schaut et Van Campenhoudt, 1994 : 84). 
Les cadres privilégiés par ces contrats étant fréquemment imposés et préconstruits par le pouvoir politique, on comprend aisément que les "nouveaux » intervenants MPS sont, aux yeux des intervenants « traditionnels », de moins en moins autonomes. En affichant clairement des « catégories à problèmes » (Toxicomanes, Immigrés, etc.), ces contrats de sécurité ne contribuent qu'à renforcer un peu plus une stigmatisation par ailleurs déjà présente. L'aide se réduit à du contrôle ou reste tout au moins ambiguë; les problèmes sont déplacés ou neutralisés mais jamais résolus. Pour les intervenants MPS «traditionnels », les problèmes sont d'ailleurs de plus en plus (re)construits en fonction des solutions disponibles. Ces solutions étant le plus souvent sécuritaires, elles servent à peine à masquer ce qui, selon eux, reste un échec face à la toxicomanie, à l'insécurité et à l'exclusion.

Il apparaît donc clair que pour le secteur de l'intervention MPS en toxicomanie, l'approche globale intégrée est un échec:

Le concept « global intégré » était bien la dernière chose à mettre en place. C'est complètement totalisant, c'est le monstre qui sous-entend que tout est confondu, que tout le monde fait tout et n'importe quoi. Or, il ne faut jamais perdre de vue qu'on est dans des compétences et des visées différentes. Tout cela ne doit bien entendu pas être nécessairement cloisonné mais au moins distingué, différencié, disjoint. Il peut y avoir des passerelles mais pas la confusion comme on la vit aujourd'hui avec le flic qui vient donner cours, le magistrat qui enjoint le prévenu d'aller se soigner, le médecin qui ne voit pas le consommateur à problèmes mais le toxicomane, etc. Il ne faut pas globaliser, il faut fédérer et il faut que chacun soit à sa place tout en ayant la possibilité d'échanger avec les autres. Il faut donc mettre les savoirs et les compétences en commun... mais sans les globaliser dans un grand machin confus (extrait d'entretien réalisé avec un intervenant MPS « traditionnel »).

Pour les intervenants « traditionnels », l'idéal n'est donc pas de « faire un » mais bien de « faire lien ». Or, l'application de l'approche globale intégrée se traduit selon eux par un «fourre-tout» engendrant confusion et absorption du socio-sanitaire par le sécuritaire. Une application qui est donc au plus loin d'une conception théorique et discursive se voulant l'apôtre $\mathrm{du}$ « faire lien ».

En effet, quel constat faisons-nous sur le terrain ? Celui d'une réorientation des concepts de globalisation et d'intégration.

Réorientation du concept de globalisation d'abord.

D'une part celle-ci insère la toxicomanie à l'intérieur de la lutte contre les drogues et la construit, sans recul, comme un problème dont la drogue 
est l'élément essentiel ou moteur. D'autre part, la globalisation aspire les multiples aspects de la toxicomanie et les multiples modalités d'intervention dans la seule orbite de la prévention de la délinquance. La politique n'est donc pas globale au sens où nous aurions pu l'espérer une prise en considération des aspects multiples de l'usage de drogues mais bien au sens où un de ces aspects prend le dessus et infiltre la logique de travail de l'ensemble des acteurs pertinents du champ délimité par la catégorie « toxicomanie » (Kaminski, $1995: 25)$.

Réorientation du concept d'intégration ensuite. Si celle-ci a bien eu lieu, c'est une fois encore, pas au sens où nous aurions pu le souhaiter. Suite aux menaces que représentaient la réduction des risques liés à l'usage de drogues et l'anti-prohibitionnisme, le monde politique a en effet intégréla réduction des risques sanitaires à la réduction des risques sécuritaires. C'est ainsi que « les formes d'action sanitaire jusqu' il y a peu intolérables - prescription de méthadone, formule d'échange de seringues - sont soudain devenues politiquement souhaitables et réalisables » (Kaminski, 1996 : 237). Bref, si l'intégration est présente, c'est d'une part, sous la bannière de l'ordre public et de la sécurité et d'autre part, sous l'autorité d'une logique sectorielle fermée, celle du ministère de l'Intérieur. Logique « dans laquelle les autres acteurs du champ social sont en effet invités à se couler dans la mesure où concepts, définitions, postulats et orientations ont été fixés préalablement par le secteur dominant » (Kaminski, $1996: 238$ ).

Si les intervenants «traditionnels » veulent se distinguer des nouveaux arrivés, ils craignent également une certaine confusion avec les policiers. Selon eux, en tablant sur une prévention «sociopolicière », le ministère de l'Intérieur aurait en effet négligé l'ambiguiité d'un tel concept. Négligence qui s'est traduite, selon les intervenants, par l'arrivée de « flics-A.S. » ${ }^{12}$ et d' "A.S. flics ». Ils reprochent par ailleurs au système pénal de dissimuler une extension du filet sous couvert d'humanisme et une confusion des rôles leur permettant d'agir au nom du sanitaire, du « social» ou du psychologique.

Cela dit, ces contrats n'auraient pas que des points négatifs. La confusion qu'ils engendrent serait aussi l'occasion de redéfinir les objectifs et les positions des intervenants MPS «traditionnels». Des positions qui semblent pourtant de moins en moins nettes dans leur rapport avec les contrats de sécurité. Opposé majoritairement à ceux-ci dans un premier temps, le secteur MPS « traditionnel » en toxicomanie se dirige en partie

12. « Assistants sociaux ». 
aujourd'hui davantage vers la négociation avec les pouvoirs publics, traduisant par là un certain pragmatisme. Ce qui était impensable hier est aujourd'hui acceptable, qu'il s'agisse de la prise en charge de la formation des nouveaux intervenants (voir ci-dessus) ou de l'élaboration de projets en commun.

Nouveaux intervenants financés par les pouvoirs publics

Les nouveaux intervenants subventionnés directement par le volet « sécurité » du plan global se sont quant à eux trouvés projetés involontairement, et parfois de façon tout à fait inconsciente ${ }^{13}$, dans un terrain franchement hostile à leur arrivée. Fantassins d'un nouveau type, ils allaient essuyer les plâtres de la culture de non-concertation des mandataires politiques et furent rapidement conduits à faire face à une réaction de rejet relativement brutale. Bien que les critiques furent avant tout orientées «sur les politiques et non sur les personnes", le discours « anticontrat de sécurité » allait avoir pour effet, nous venons de le voir, de créer une nouvelle image de travailleur social, celle de « l'assistant-socialau-service-de-la-police » dont l'intervention s'apparente au renseignement et à la délation. Très vite, nombre de ceux-ci estimèrent cette image inacceptable et incompatible avec leur propre déontologie et décidèrent de contrer la critique, notamment par l'affirmation de leur compétence et de leur éthique propre et par l'adoption d'une attitude de transparence quant à leurs méthodes de travail. C'est ainsi que le secteur connut une période riche en « chartes ", en contrats de collaboration ou en conventions de toutes sortes visant à amenuiser la méfiance des intervenants « traditionnels » et à construire la spécificité — et l'honorabilité — du champ spécifique d'intervention des nouveaux arrivés.

S'ils sont diabolisés par le secteur «traditionnel », ces nouveaux intervenants insistent par ailleurs sur la nécessité de leur mise en place, témoin cet extrait d'entretien avec un membre du personnel du centre Transit :

13. Il est important de souligner que la politique d'embauche (et de clientélisme...) souvent à l'œuvre dans les communes a favorisé l'engagement de travailleurs criminologues, assistants sociaux ou éducateurs de rue relativement jeunes et souvent inexpérimentés. Engagés dans le cadre de contrats relativement précaires (durée déterminée, temps partiel...), une grande partie du personnel de prévention semblait être constitué de « premiers emplois » pleins de bonne volonté mais bien souvent dépourvus du bagage professionnel nécessaire à leur mission. 
Quand vous êtes face à des situations où une personne qui n'a ni ressources financières, ni mutuelle, ni carte d'identité doit rentrer à l'hôpital mais ne sera acceptée qu'en ordre administrativement, je ne vois pas, à part nous, qui va s'en occuper. Par ailleurs, les besoins auxquels nous devions répondre se sont élargis très rapidement avec l'accueil croissant de gens exclus des centres thérapeutiques résidentiels et des milieux hospitaliers. Des cas difficiles donc mais comme l'accueil de Transit était ouvert à tous, il n'y avait aucune raison de ne pas accepter ceux-ci. Bref, s'il est vrai que Transit constitue un intermédiaire entre la rue et les institutions, il faut reconnaître que bien souvent, il constitue aussi un intermédiaire entre les institutions et la rue.

Ces nouveaux intervenants estiment ne pas travailler « sous contrainte " ni même avoir une finalité sécuritaire et ce, pour deux raisons : a) s'ils reconnaissent contribuer accessoirement à la sécurité de la ville, ils insistent sur le fait que là n'est pas leur finalité directe. La sécurité ne serait dès lors qu'un des effets positifs de leur objectif premier, à savoir, pour Transit, un accueil d'urgence et une remise en ordre socio-administrative; b) il n'y a rien qui filtre entre leur service et la police. Ils rappellent d'ailleurs que, si c'était le cas, il est clair qu'il leur deviendrait rapidement impossible de travailler. Bref, leur perception témoigne d'un détachement par rapport à la dimension symbolique de leur financement. Ce personnel semble en effet peu préoccupé par le ministère qui le finance. C'est davantage la dimension « service public » que la dimension sécuritaire qui, dans leur mission, pose problème : rigidité; encodage de données souvent superflues; immobilisme; formalisme; manque de flexibilité; primauté du travail administratif sur le travail social... S'ils n'en paraissent pas conscients, d'autres facteurs témoignent cependant d'une contrainte liée à leur inscription sinon sécuritaire, à tout le moins gestionnaire : désagréable impression de privilégier les moyens (du bord) sur les fins; sentiment de devoir " invisibiliser » les publics jugés menaçants tout en « visibilisant » au maximum les actions menées à leur égard, impression de provoquer la demande, etc.

\section{Policiers communaux}

La police communale, tout comme les intervenants nouvellement arrivés, fut amenée à faire face à la critique prononcée des membres du secteur déjà en place. Accusée de tirer tous les bénéfices de la réforme et de tenter de brouiller les pistes par l'introduction d'une « nouvelle culture de collaboration » dont elle aurait seule le profit, la police allait se retrouver au centre de débats houleux initiés par les intervenants du secteur 
« traditionnel ». L'historique des rapports entretenus entre ces derniers et les forces de l'ordre permet cependant de comprendre le peu d'impact que semble avoir eu sur les policiers de terrain cette réaction de rejet et d'indignation. De tout temps en effet, les rapports entre les forces de l'ordre et les intervenants médico-psycho-sociaux (particulièrement en ce qui concerne le secteur de la toxicomanie) se sont caractérisés par une grande tension aboutissant la plupart du temps à une ignorance réciproque et une volonté de départager le plus nettement possible les rôles et les secteurs d'intervention. La mise en débat suscitée par l'arrivée de nouvelles politiques publiques n'allait constituer pour les forces de l'ordre, qu'un mouvement d'opposition de plus, ne nécessitant pas de prise de position particulière et ne déstabilisant aucunement leur mode de fonctionnement.

Par ailleurs, nous avons constaté sur le terrain policier, une relative ignorance de ce que pouvaient apporter comme ressources ces nouveaux dispositifs. Ainsi, l'arrivée des contrats de sécurité, loin de susciter l'enthousiasme, allait à son tour entraîner la polémique à l'intérieur des services de police. L'allocation et la répartition des moyens et des ressources, on le sait, constituent dans toute institution un point de conflit, et ce n'est pas la police qui allait échapper à ce type de problème.

Les contrats de sécurité, c'est magouille et compagnie ! OK, il y a de l'argent distribué. Mais on ne sait pas vraiment où celui-ci va. On a bien créé certains services qui pourraient être utiles... Mais comme toujours on n'a pas consulté la base et on a été très peu informé... et beaucoup de cet argent est utilisé sans beaucoup de bon sens. Alors moi, ce que j'en pense... (extrait d'entretien réalisé avec un agent de police communale travaillant dans une section non spécialisée).

Le seul dispositif très clairement établi en réponse à une demande policière, le centre résidentiel Toxico-Transit pour usagers de drogues interpellés par la police, allait quant à lui rapidement changer d'orientation faute de clientèle, les policiers lui ayant en définitive très peu amené d'usagers de drogues: un paradoxe imputable au manque d'intérêt des policiers de base pour un dispositif mis en place à la demande de la hiérarchie. De centre « au service de policiers dépourvus de réponse ", Transit allait, rappelons-le, devenir un centre au fonctionnement somme toute assez classique, c'est-à-dire travaillant à la demande de l'usager lui-même ou en relais avec d'autres institutions.

Du reste, la volonté des travailleurs sociaux engagés par les pouvoirs publics de se créer une légitimité auprès de leurs collègues « traditionnels » s'est souvent traduite par une prise de distance assez 
radicale à l'égard des forces de l'ordre. Une position qui allait avoir pour effet de susciter la confusion dans l'esprit des policiers de terrain. Assimilant les nouveaux travailleurs sociaux aux anciens, ceux-ci n'ont pas vu dans les travailleurs des contrats de sécurité de nouveaux partenaires privilégiés. Au contraire, ce mécanisme de protection eut pour effet de persuader un certain nombre de policiers que les contrats de sécurité ont, à leur défaveur, renforcé les moyens des acteurs sociaux concurrents (voire " ennemis») intervenant sur leur propre terrain. Le modèle d'opposition du « répressif » contre « le social » allait dès lors se trouver renforcé, court-circuitant toute possibilité d'alliance objective entre les deux secteurs.

Quels constats retirer des points de vue de ces trois catégories d'acteurs? Il apparaît assez évident que si les différents discours se recoupent ici et là, ils ne se confondent aucunement. Pour les intervenants " traditionnels", leurs nouveaux homologues et les policiers communaux ne font qu'un et il n'est aucunement question d'assimiler les formes de travail social « à vocation sécuritaire » à leur propre intervention. Pour les nouveaux travailleurs MPS, il importe, tout en se reconnaissant peu innovants, de se distinguer des forces de l'ordre en vue d'être rapidement associés au secteur « traditionnel ». Pour les policiers enfin, nouveaux et anciens intervenants MPS ne font qu'un. On comprend, au vu de ces résultats, qu'il est difficile de parler de la mise en place d'un "faire lien ». Ceci nous conduit à souligner les paradigmes en présence, renvoyant tantôt à la fusion par l'excès d'assimilation tantôt à l'opposition. On est donc loin de la logique d'alliance espérée. Un autre constat fait suite aux entretiens réalisés, celui d'une approche toujours plus défensive de la gestion du social. Bref, nous serions aujourd'hui dans une logique de "défense sociale ${ }^{14}$ et non, comme on pourrait le souhaiter, de « protection sociale $»^{15}$.

14. La défense sociale « est fondée sur l'idée que la société doit être défendue de ceux de ses membres qui la menacent par leurs comportements asociaux. Ces «marginaux » ne peuvent bénéficier d'une assistance ou de la suspension d'une sanction que s'ils se plient à une tutelle correctrice. Dès lors, la forme d'intervention sociale qu'adopte la défense sociale couple l'assistance et la répression » (Schaut et Van Campenhoudt, $1994: 82$ ).

15. La protection sociale, elle, "repose au contraire sur l'idée que les individus en situation précaire ou problématique sont victimes des dures lois de la société, de ses inégalités et des mécanismes d'exclusion qui y ont cours. Les bénéficiaires des interventions sociales sont donc des personnes à priori "normales" qui ont automatiquement droit, à titre d'affiliés, à la sécurité sociale » (Schaut et Van Campenhoudt, 1994 : 82). 


\section{Défense sociale et centralité de la notion de risque}

Notre État s'apparente de plus en plus à un «"État de prévention" obsédé par la nécessité d'anticiper tout danger potentiel et de déplacer les seuils de l'intervention pénale vers des situations antérieures à l'acte » (Cartuyvels et al., 1997 : 231). Les politiques de prévention passeraient en effet de l'action sociale au contrôle social. Et, si hier la prévention aidait et transformait, elle ne visera plus aujourd'hui « qu'à gérer, réduire ou contrôler des situations à risques» (Cartuyvels et al., 1997 : 230). Alors que le pouvoir politique parlait d'associer une prévention soucieuse de s'attaquer à l'exclusion sociale à une prévention situationnelle et sécuritaire, la première est restée en fin de compte cantonnée au discours d'intention. La seconde, par contre, sera florissante dans la mesure où "se développe un potentiel de personnes qui doivent faire l'objet d'un contrôle renforcé, puisque exclues du marché de travail, source de discipline » (Albrecht, 1997 : 127).

Même si bon nombre d'intervenants «traditionnels » continuent à lutter pour préserver leur autonomie, ces récentes politiques publiques n'en symbolisent pas moins une nouvelle façon, dominante, de gérer le social sur un mode défensif. Cette nouvelle gestion du social, nous la devons à une nouvelle manière de penser le politique. Celle-ci est en effet le fruit « du déclin de la rationalité téléologique propre à l'interventionnisme de l'État social » (Cartuyvels, 1996b : 592). Fruit aussi de la fin du rêve intégrateur que prônait ce dernier. Et « s'il n'est plus tant question aujourd'hui de social que de solidarité, d'intégration que d'insertion, d'émancipation que d'accompagnement, de traitement que de réduction des risques, de socialisation que de contrôle social, c'est bien que l'idéal d'une action transformatrice en profondeur recule devant un souci plus pragmatique de gestion du statu quo ou de contrôle des effets problématiques de la nouvelle "question sociale" (Cartuyvels, 1996b : 592).

Associées, normes économiques et normes sécuritaires promeuvent dès lors une approche défensive de la gestion du social mais aussi une approche pragmatique du traitement pénal où « l'important ne serait pas tant de réhabiliter les délinquants que de minimiser leurs gains, maximiser leurs coûts et, si nécessaire, neutraliser ceux dont l'activité criminelle est trop virulente » (Cusson, $1988: 433$ ).

Bref, qu'elles soient « sociales » ou sécuritaires, ces nouvelles politiques publiques ne répondent donc pas à la nécessité actuelle de repenser les rapports entre acteurs sociaux et institutions. Refusant ou étant inca- 
pables de penser à long terme, elles se reconnaissent de moins en moins une dimension d'utopie progressiste et doivent sans doute davantage s'interpréter comme une réponse aux impératifs de l'urgence.

\section{Conclusions}

À travers l'exemple de la politique belge en matière de sécurité et d'usage de drogues, nous avons tenté d'interroger empiriquement la pertinence d'une expérience qui se voulait novatrice. Cette «nouvelle façon de penser la sécurité », prônée par le ministère de l'Intérieur, poursuivait comme ambition d'intégrer réponse sociale et réponse sécuritaire, notamment en matière d'usage de drogues. La résistance des acteurs de terrain au modèle proposé par les pouvoirs publics nous conduit à présent à considérer celui-ci de façon critique.

Pour bien saisir la différence entre la conception théorique d'une approche globale intégrée et sa mise en place sur le terrain, nous avons fait appel à la distinction de Durand (1992) entre imaginaire de fusion et imaginaire d'alliance. Alors que le premier est régi par des mots comme « inclure », « absorber » ou encore " intégrer », le second, lui, sera caractérisé par les mots "relier», "dialoguer », "créer des relations ». Si l'imaginaire de fusion suivra une logique syncrétique et cherchera ce qui se ressemble, l'imaginaire d'alliance suivra une logique de relation dialectique et cherchera ce qui relie les antagonismes. Malgré leur parenté, imaginaires d'alliance et de fusion se distinguent donc d'une manière capitale. Si l' "alliance " vise à articuler une dualité, elle ne cherchera pas à la confondre. Elle n'est ni métissage, ni amalgame mais bien articulation dialectique d'identités distinctes: elle reconnât les différences, admet les oppositions, souligne les aspérités mais vise au mouvement qui les relie et les allie. Or, s'il est bien une chose que nous avons pu observer sur le terrain, c'est une sérieuse crainte, de toutes parts, de voir s'imposer un modèle de travail en commun fondé davantage sur la fusion (voire de l'absorption...) que sur l'alliance. La crainte de voir la sphère pénale s'agrandir et absorber le reste fut notamment à la base de nombreux discours, y compris celui des nouveaux travailleurs engagés par les pouvoirs publics, travailleurs pourtant censés faire le lien entre les diverses approches coexistant sur le terrain.

Qu'en est-il alors de la possibilité d'associer, dans les mêmes dispositifs, une option sécuritaire reposant sur un fondement pénal — ou répressif - avec une approche médico-psycho-sociale émancipatrice 
(et donc, de créer au passage un nouveau champ d'intervention hybride) ? Doit-on à priori diaboliser une telle synergie ? Peut-on allier sécurité et solidarité ? Est-ce que donner plus de place au champ sécuritaire induit nécessairement un investissement moindre dans les politiques sociales?

Pour certains, une telle alliance est irréalisable, dans la mesure où dans ce cas, l'approche socio-sanitaire serait absorbée et instrumentalisée à des fins répressives ou au mieux managériales. Cette position qui diabolise toute connotation sécuritaire peut se justifier et semble d'ailleurs parfaitement traduire ce qui s'est passé sur le terrain observé. D'un autre côté, on peut difficilement soutenir la position de la « chasse gardée » (clientèle médico-psycho-sociale vs clientèle pénale). Cette logique réduit en effet la gestion des problèmes sociaux à une répartition administrative des compétences (gestion policière répressive des «irrécupérables » et gestion médico-psycho-sociale adoucie des « recyclables »). Si cette distinction a l' « avantage » de conforter nos catégories, elle fatalise parallèlement les associations entre police et répression et entre intervenants médico-psycho-sociaux et prévention. La question devient alors « quelle place laisser à la sécurité en sachant qu'elle diminuera de facto la place prise par la solidarité ? ». Bref, cette question est mal posée, dans la mesure où elle n'interroge que le problème de la place alors que c'est surtout la forme que prend cette sécurité qui, selon nous, est importante. Dans la mesure aussi - mais il faut être très prudent — où plus de sécurité, si cela est bien pensé, peut aussi engendrer plus de liberté.

\section{Références}

Albrecht, P. A. 1997. "La politique criminelle dans l'État de prévention » Déviance et société 21 (2) : 123-136.

CARTUYvels, Y. 1996a. «Insécurité et prévention en Belgique : les ambiguiités d'un modèle 'global-intégré' entre concertation partenariale et intégration verticale » Déviance et société 20 (2) : 153-171.

CartuYvels, Y. 1996b. «Les politiques de prévention socio-pénales en Belgique, métamorphose de l'action étatique?» Pp. 581-603 in Droit négocié, Droit imposé, sous la direction de P. Gérard, F. Ost et M. Van de Kerchove. Bruxelles : FUSL.

CARTuYvels, Y. et MARY, P. 1997. "Crise de la Justice : et au-delà ? » Pp. 97126 in L'affaire Dutroux. La Belgique malade de son système, Collectif. Bruxelles : Éditions Complexe. 
Cartuyvels, Y., Digneffe, F. et Kaminski, D. 1997. "Droit pénal et déformalisation », Pp. 219-242 in Les mutations du rapport à la norme. Un changement dans la modernité, sous la direction de J. De Munck et M. Verhoeven. Bruxelles : De Boeck.

Cusson, M. 1988. « La criminologie a-t-elle un avenir ?» R. I. C. P. T. XLI (4) : 428-436.

DEVRESSE, M.-S. 1998. "Les contrats de sécurité, contrats ou contraintes ? » Agenda interculturel (162) 20-23.

DEVRESSE, M.-S. et GuILLAIN, C. 1996. « Les nouvelles politiques socio-pénales en matière de toxicomanie » Les Cabiers de prospective jeunesse 1 (1-2) : 42-48.

DuRAND, G. 1992. Les structures anthropologiques de l'imaginaire, Paris : Dunod.

KAMINSKI, D. 1995. "Quelques changements dans l'indifférence " Bruxelles Informations sociales (134-135) : 23-26.

KAMINSKI, D. 1996. «Approche globale et intégrée : de l'usage politique des drogues » Pp.233-242 in Approches sécuritaire et socio-sanitaire : complémentaires ou contradictoires ?, sous la direction de B. De Ruyver, G. Vermeulen, A. De Leenheer et Th. Marchandise. Actes du IIIème congrès national « Gestion des drogues en $2000 »$. Antwerpen/Bruxelles : Maklu/Bruylant.

SCHAUT, C. et VAN CAMPENHOUDT, L. 1994. Le travail de rue en communauté française, Bruxelles : Fondation Roi Baudouin. 